\title{
INFLUENCE OF CHANGING AMPLITUDE OF INITIAL IMPERFECTIONS ON LATERALLY LOADED CYLINDRICAL SHELL
}

\author{
Ondrej Voltr \\ University of Pardubice, Czech Republic \\ ondrej.voltr@upce.cz
}

\begin{abstract}
The article is devoted to the research of thin-walled shell structures. Specifically, this article deals with the influence of initial imperfections on the loss of stability of the laterally loaded cylindrical shell. Previous contributions of the author on this topic were using initial imperfections of constant amplitude; however, initial imperfections of changing amplitude were used in this phase of research. The examined structure is considered as a part of the simplified case of the cylindrical shell (part of a road tank), which is located on saddle supports. The beginning of the initial shape imperfections is considered as state of overloading of the structure from its own weight and the weight of its content. The initial imperfections of the shape are created by pushing the rigid saddle support into the shell. Therefore, the new deformed shape of the shell can be used as an imperfect shell in the following nonlinear analysis. The objective of the analysis is to evaluate the socalled reduction factor $\alpha$. The reduction factor depends on the change of the geometric parameters of the numerical model and on the change of amplitude of initial imperfections. The most important geometric parameters are the embracing angle of the saddle support $2 \theta$ and the wall thickness of the shell t. The changing amplitude of the initial imperfections $\Delta \mathrm{w}$ is represented here by the dimensionless parameter $\Delta \mathrm{w} / \mathrm{t}$ with regard to the shell thickness t. The numerical analysis was carried out by means of a computer program COSMOS/M based on the finite element method (FEM).
\end{abstract}

Keywords: shell, imperfections, stability, FEM, saddle.

\section{Introduction}

This article focuses on thin-walled shell structures. The investigated structure is based on a cylindrical road tank located on saddle supports (for example [1]). Therefore, a laterally loaded cylindrical shell as the form of a simplified case of the cylindrical shell with rigidly attached saddle support (saddle) is investigated [2].

Such structures - saddle supported cylindrical shells - are solved, for example, in the European Design Recommendation ECCS [3], where the method determined by V. Krrupka [4;5] is used. The influence of initial imperfections on the stability of cylindrical shell under few types of loading (axial compression, bending moment, torque moment, etc.) is taken into consideration in the ECCS [3]. But not specifically for the saddle supported cylindrical shell. For the first approach it was the only available comparison, hence the probably conservative. Actually, the influence of initial imperfections on the stability of saddle supported cylindrical shells was not yet directly examined. Already

known is that with the initial imperfection is introduced an additional bending state into the shell [6], which usually decreases carrying capacity of the shell [7;8]. The influence of initial imperfections is expressed by the so-called reduction factor $\alpha$. So, motivation for this research is to explore and determine the influence of initial imperfections on the loss of stability of the laterally loaded cylindrical shell specifically.

For that purpose a numerical nonlinear analysis based on the finite element method and performed by computer program COSMOS/M [9] serves. Following the article [2] there were performed numerical analyses on models with commonly used variants of the embracing angle $2 \theta\left(60^{\circ}, 90^{\circ}\right.$ and $120^{\circ}$ ) and the range of the thin-walled parameter $R / t<68 ; 250>$. The $R / t$ parameter is equal to the ratio of the shell radius $R$ and the thickness of the shell $t$. The analyses monitor changes of the reduction factor $\alpha$ with changing parameters of the numerical model. During the loading attention is also paid to the character of the deformation of the shell. The loss of stability is solved only in the elastic range.

One important parameter differs from the previous work [2] - amplitude of depth of initial imperfection $\Delta w$. The first phase of the research considered the amplitude $\Delta w$ as constant. This next phase considers it with regard to the shell thickness and introduces a dimensionless parameter of changing amplitude of initial imperfection $\Delta w / t$, which varies in ratios $0.1,0.5,0.75,1.0,1.5,2.0$. So, 
the current phase of the research examines the influence of initial imperfections of changing amplitude $\Delta w / t$ on the loss of stability of laterally loaded cylindrical shells.

\section{Numerical model}

The basis of the numerical model (see Fig. 1) is the thin-walled cylindrical shell with an invariable length $L=300 \mathrm{~mm}$, an invariable radius of the shell $R=75 \mathrm{~mm}$ and the thickness $t$, which varies in the range $0.3 \div 1.1 \mathrm{~mm}$ in increments of $0.1 \mathrm{~mm}$ [2]. This shell has at both ends flat thick circular covers with the thickness $t_{1}=30 \mathrm{~mm}$. The thickness ensures sufficient rigidity of the covers. Also, they prevent their excessive deformation and help avoid negative influence to the analyzed results. In the middle of the shell the rigid punch in the shape of a saddle support with a width $b=20 \mathrm{~mm}$ and embracing angle $2 \theta\left(60^{\circ}, 90^{\circ}, 120^{\circ}\right)$ is firmly connected to the cylindrical shell. Fig. 2 shows the example of the numerical model in the variant of initial undeformed model without initial imperfection for embracing angle $2 \theta=120^{\circ}$.

A combination of simple support in two edge nodes of finite element mesh (points A, B see Fig. 1) and prevention of the saddle support from lateral displacements and tilting was chosen as boundary condition. The shell may thus lose stability in both the symmetrical and the nonsymmetrical shape according to the vertical plane passing through the axis of the cylindrical shell. Vertical force $F$ is introduced into the numerical model through the saddle.

The material of all parts of the model (shell, covers, saddle support) has the same mechanical properties, particularly the Young modulus of elasticity $E=1.9 \mathrm{E}+5 \mathrm{MPa}$ and Poisson's ratio $\mu=0.3$.

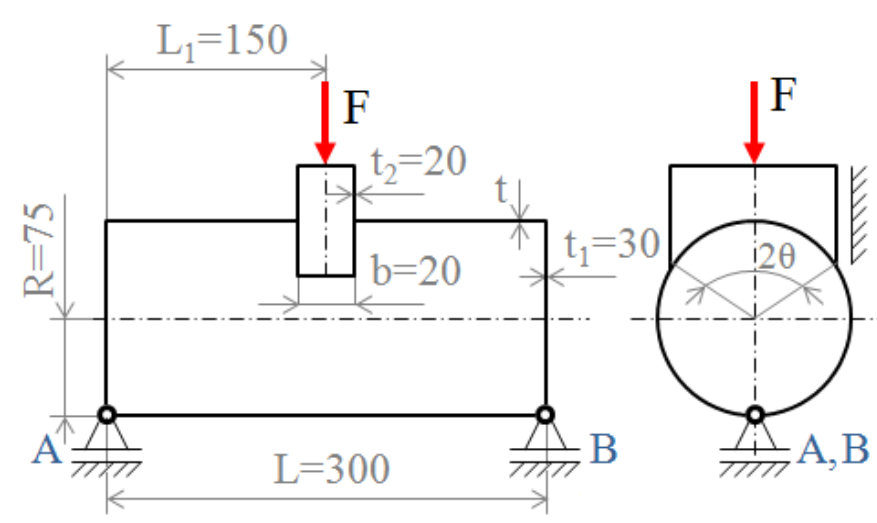

Fig. 1. Geometric parameters of numerical model

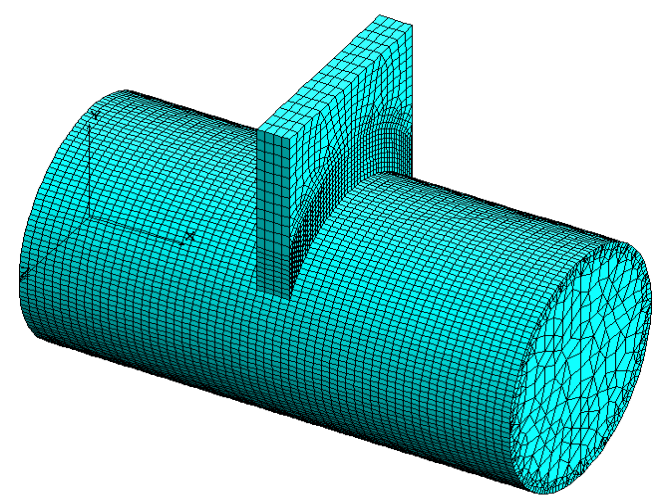

Fig. 2. Example of initial undeformed model $\left(2 \theta=\mathbf{1 2 0}^{\circ}\right)$

\section{Results and discussion}

The typical course of deformation of the assessed laterally loaded cylindrical shell is described. Due to the fact that the course of deformation is approximately the same for all listed embracing angles, it will not be mentioned again in the result section of any individual embracing angles. It should be noted that the deformation scale of the following deformed numerical models is enlarged for better visualisation and the understanding of the process of the loss of stability.

In the first phase of loading, the saddle is gradually pushed into the shell (Fig. 3). Deformation of the shell occurs in a symmetrical shape with well visible changes in the form of waves. Waves have the maximum deflection in the vicinity of the saddle and gradually diminish towards the shell ends. The nature of the waves differs slightly for each embracing angle.

In the second phase, the cylindrical part of the shell tilted to the side (Fig. 4). The shell has a tendency to lay on one edge of the saddle. The consequence of the tilting is the redistribution of the previously formed waves into a new shape. On the tilted side of the shell the deformation is significant, but on the other side the deformation begins to decrease.

The last phase can be described as a partial "smoothing" of deformations from the previous two phases (Fig. 5). This results in a significant deformation near the saddle on the tilt side of the shell. 
While on the other side of the saddle, the deformation is almost completely eliminated. For comparison, the sample examples are shown side by side in Fig. 3, Fig. 4 and Fig. 5.

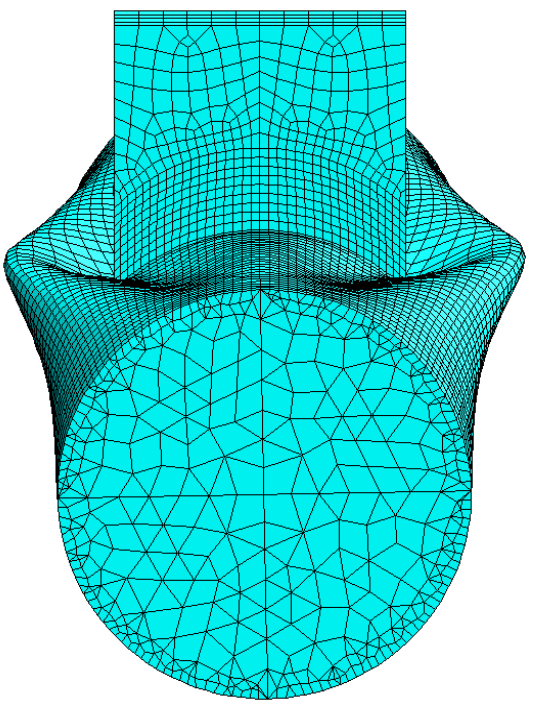

Fig. 3. First phase of deforming pushing of the saddle

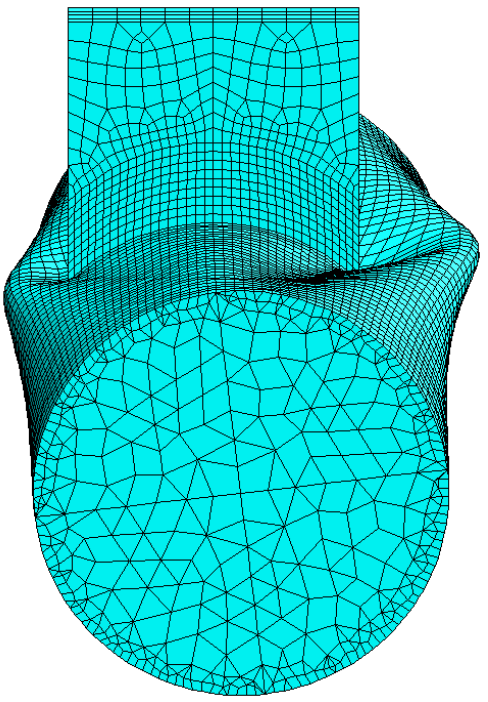

Fig. 4. Second phase of deforming - side tilting

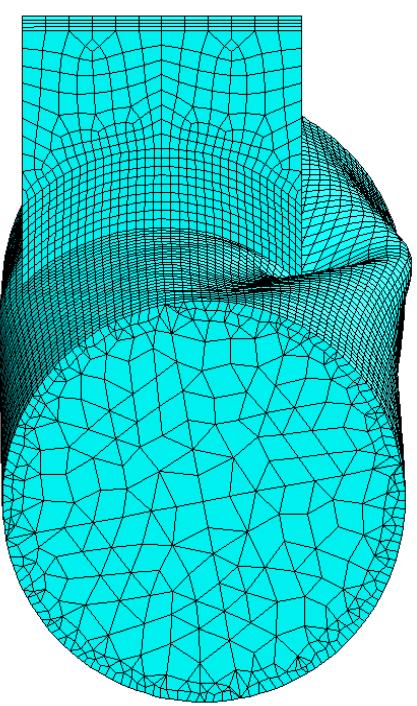

Fig. 5. Third phase of deforming - smoothing

The described course of deformation is similar to perfect and imperfect shells. For imperfect shells, smaller local deformations in the vicinity of the saddle occur due to the initial imperfection.

For most variants of models, it can be identified as significant loss of stability associated with the transition from the first to the second phase of deforming.

\section{Results of embracing angle $2 \theta=120^{\circ}$}

The outputs of the performed numerical analysis are the curves of the reduction factors $\alpha$. For each variant of the thin-walled parameter $R / t$ and $\Delta w / t$ parameter nine curves (Fig. 6) are plotted. In all observed cases, the curves of the reduction factors have decreasing character with the increasing $\Delta w / t$ ratio. The effect of the initial imperfection progressively increases with increasing the ratio $\Delta w / t$ and the values of the reducing factor $\alpha$ therefore decrease. However, the maximal decrease is $14 \%$, which compared to an ideal shell corresponds to $\alpha=1$.

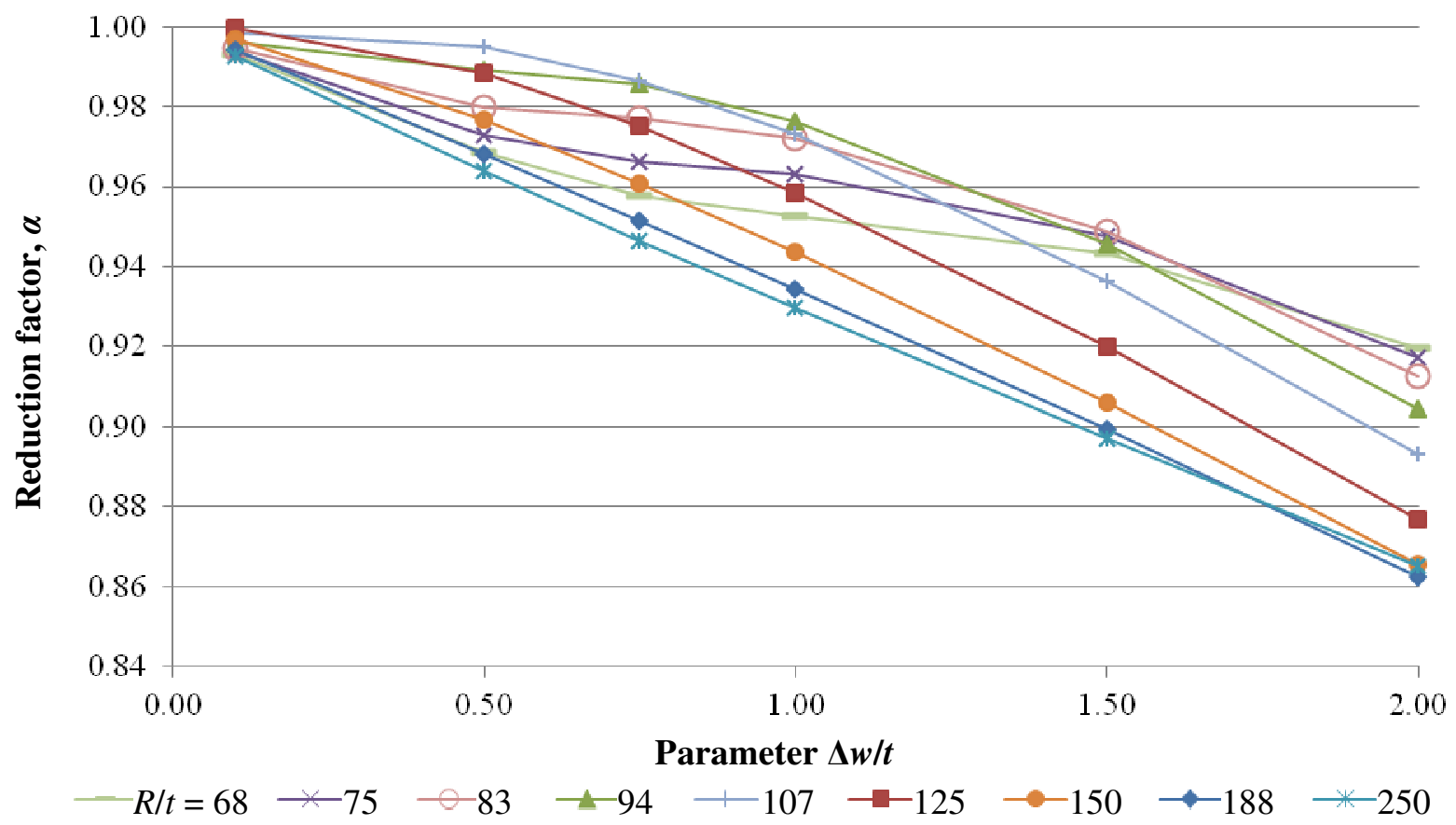

Fig. 6: Results for variant with embracing angle $2 \theta=120^{\circ}$ 
The results show that in the most thin-walled variants $(R / t=125-250)$ it is possible to observe the symmetrical shape of the deformation of the shell almost or even directly until the loss of stability. However, regardless of the ratio of $\Delta w / t$ the shell is tilted to the side after the loss of stability. With decreasing the thin-walled parameter (for $R / t=68-107$ ), this direct continuity disappears and the cylindrical part of the shell tilted regularly close before the loss of stability. However, the effect on the nature of the curves is minimal regardless the value of the $R / t$ parameter and the curves are very similar (see Fig. 6).

\section{Comparison of results of embracing angles $2 \theta=90^{\circ}$ and $2 \theta=60^{\circ}$ with $2 \theta=120^{\circ}$}

To illustrate the results of the model variants $2 \theta=90^{\circ}$ and $2 \theta=60^{\circ}$ there are in Fig. 7 and Fig. 8 plotted graphs showing the dependence of the reducing factor $\alpha$ on the parameter $\Delta w / t$. In both cases, the graphs show results of nine variants of thin-walled parameter $R / t$.

When comparing the results of variations of the embracing angle $2 \theta=90^{\circ}$ in the graph of Fig. 7 to the previous variant $\left(2 \theta=120^{\circ}\right.$ in Fig. 6), a similar character of curves of reducing factors $\alpha$ can be seen. With the increasing parameter $\Delta w / t$ the decreasing nature of the curves $\alpha$ is noticeable and this decrease is steeper than the variant $2 \theta=120^{\circ}$. None of the reduction factor values falls below $\alpha=0.89$ (when the lowest value for $120^{\circ}$ was $\alpha=0.86$ ). In one case of the thinnest variant $R / t=250$ (for $\Delta w / t=0.5)$ the curve of the reduction factor $\alpha$ is even slightly less than the value $\alpha=1$. However, comparing this course of deformation with the other related courses, there was not seen any specific reason for this slight increase. Furthermore, it is possible to observe the interesting situation for $\Delta w / t=2.0$, where relatively close match of values occurred across the range of thin-walled parameters $R / t$. A similar match was only at variant $\Delta w / t=0.1$. Overall, the initial imperfection causes a decrease of a maximum of $11 \%$ as regards to the value of the shell without initial imperfection. By comparing the load curves and the deformation patterns of the models, it has been shown that in most cases the shell lost stability relatively soon after the transition from the linear to non-linear part of the loading curve.

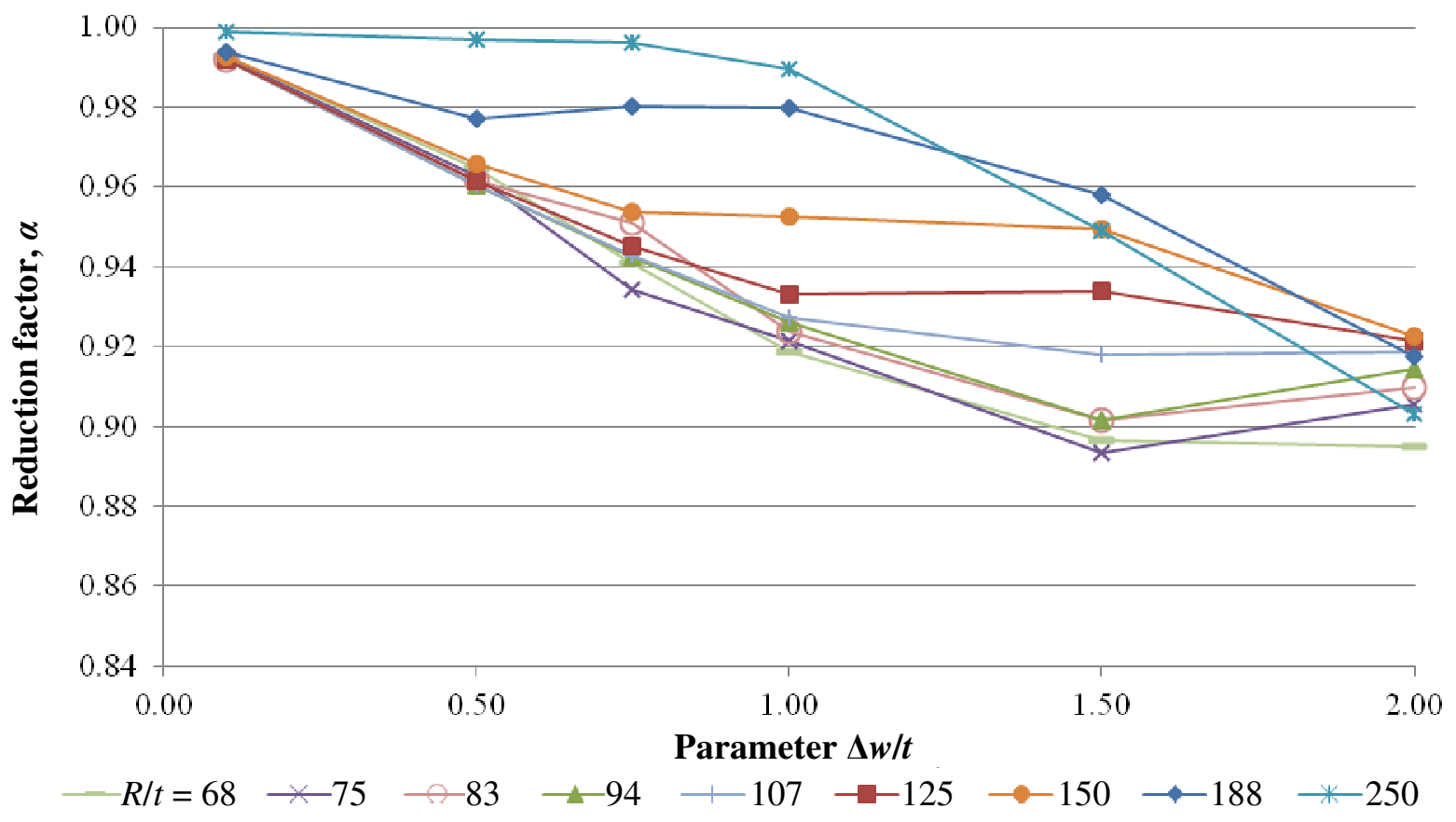

Fig. 7: Results for variant with embracing angle $2 \theta=9^{\circ}$

Fig. 8 shows the reduction factor curves of variant $2 \theta=60^{\circ}$, and the differences versus variant $2 \theta=120^{\circ}$ can be seen here. The nature of the reduction factor curves $\alpha$ differs from the very beginning. The curves are not completely consistent either with the values of $\alpha$ for the minimum amplitude of imperfection $(\Delta w / t=0.1)$. Firstly, the trend is a steeper decrease in the reduction factor curves $\alpha$, in two cases below $\alpha=0.9$ limit $(R / t=68$ and 75). Only in the last section $(\Delta w / t=1.5 \div 2.0)$ the curves are characterized by a slight increase. The course of the $R / t=188$ variant is slightly higher 
than the remaining curves. Two variants from the opposite end of the range of $R / t(R / t=68$ and 75$)$ are the second interesting contrast. In both cases, these are the variants that were most affected by the initial imperfection and reached the lowest values of $\alpha$. For variants $60^{\circ}$ and $90^{\circ}$, this is exactly the opposite of variant $120^{\circ}$. These variants of $R / t$ for $120^{\circ}$ reached almost always the highest values of the reduction factor $\alpha$ (influence of the initial imperfection was the lowest).

For both variants of the embracing angle, the effect of the initial imperfection in the overall view seems to be slightly higher than for the variant $2 \theta=120^{\circ}$. This is evidenced by a steeper decrease in the reduction factor values, including fractional minimum values.

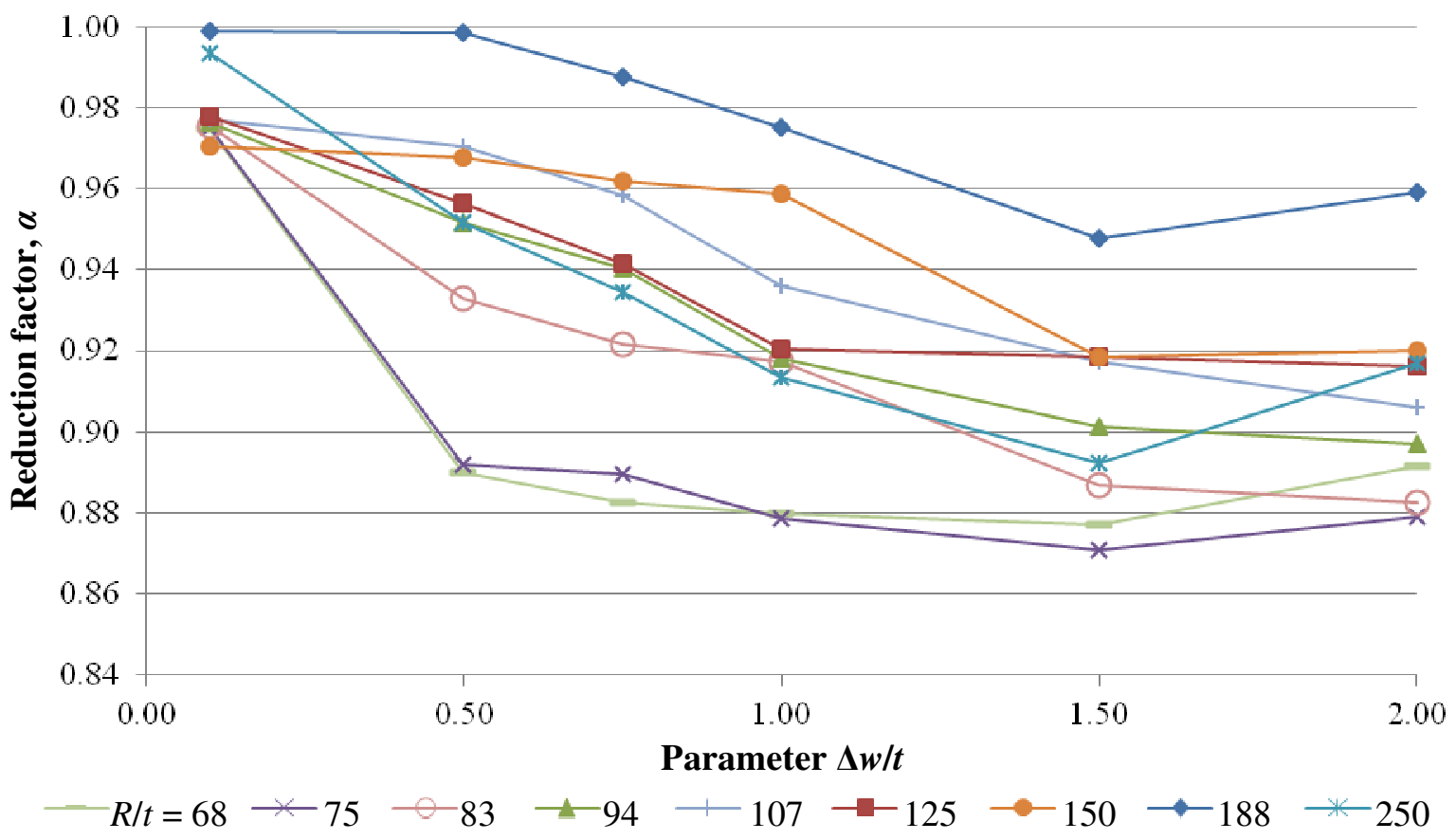

Fig. 8: Results for variant with embracing angle $2 \theta=60^{\circ}$

\section{Conclusions}

The paper examines the influence of different values of the initial imperfection amplitude on the loss of stability of the laterally loaded cylindrical shell. After the detailed evaluation of the individual numerical analyzes, the outputs represented by the load curves, the shapes of the deformation of the shell and the values of the reduction factor $\alpha$, the following conclusions can be drawn:

For variants with the embracing angle $2 \theta=120^{\circ}$, the results shown in Fig. 6, through the dependency of the reduction factor $\alpha$ and the parameter of amplitude of initial imperfections $\Delta w / t$. The resulting curves of the reduction factor $\alpha$ are very similar for all variants of the thin-walled parameter $R / t$. The maximum difference between the values of the highest and the lowest curve is around $6 \%$. Without exception, all curves decrease over the entire range of $\Delta w / t$ and at the end of this range reach the lowest values of the reduction factor $\alpha$ for all variants of the embracing angles.

Variants of embracing angles $2 \theta=60^{\circ}$ and $2 \theta=90^{\circ}$ differ mainly in the faster decrease of the reduction factor $\alpha$ value. In most cases the decrease stopped at $\Delta w / t=1.5$; after that slight increase followed. Except for a few exceptions, the individual curves are relatively close, with similar courses of the curves. The results of variants $2 \theta=90^{\circ}$ and $2 \theta=60^{\circ}$ are shown in Figs. 7 and 8 .

In all observed cases, initial imperfection caused decrease in the carrying capacity of the shell by maximum of $14 \%$ (for $2 \theta=120^{\circ}$ ), respectively $11 \%$ for $2 \theta=90^{\circ}$ and $13 \%$ for $2 \theta=60^{\circ}$. Therefore, from the decreases shown in the analyses the influence of initial imperfections seems rather insignificant.

\section{Acknowledgements}

This work was supported by the University of Pardubice within the project No. SGS_2018_023. 


\section{References}

[1] Paščenko P., Stejskal P. Cisterna NKA 46. Kontrola pevnosti - podklad k přípravě měření při jízdních zkouškách (Road tank NKA 46. Strength analysis - preparation of measurement in driving tests. Developmental report of AK-Mechanika, s.r.o.), 2008, Pardubice, Czech Republic. (in Czech).

[2] Voltr O., Paščenko P. Initial imperfections of horizontal cylindrical shells on saddle supports. Proceedings of 30th International Colloquium on "Advanced Manufacturing and Repair Technologies in Vehicle Industry”, May 22-24, 2013, Visegrád, Hungary, pp. 77-82.

[3] Rotter J.M., Schmidt H. (Eds) Buckling of Steel Shells: European Design Recommendations, Fifth edition. Published by ECCS, 2008. 384 p.

[4] Křupka V. Buckling and limit carrying capacity of saddle loaded shells. Proceedings of an International Colloquium on "Stability of Plate and Shell Structures", April 6-8, 1987, Ghent, Belgium, pp. 617-622.

[5] Křupka V. Buckling and plastic punching of circular cylindrical shells due to saddle or lug loads. Proceedings of an International Colloquium on "Buckling of Shell Structures, on Land, in the Sea, and in the Air", September 17-19, 1991, Lyon, France, pp. 11-20.

[6] Volmir, A.C. Ustojčivost' uprugich sistěm. (Stability of the elastic system). Moskva: Gosudarstvennoe izdastělstvo fiziko-matematiceskoj literatury, 1963. (In Russian).

[7] Wunderlich W., Deml M. Direct evaluation of the worst imperfection shape in shell buckling. Computer Methods in Applied Mechanics and Engineering Journal, vol. 149, 1997, pp. 201-222.

[8] Wunderlich W., Albertin U. Analysis and load carrying behavior of imperfection sensitive shells. International Journal for Numerical Methods in Engineering, vol. 47, 2000, pp. 255-273.

[9] FEM Computer program COSMOS/M, version 2.95, 2010. 